\title{
INTEGRATION OF SHIP HULL ASSEMBLY SEQUENCE PLANNING, SCHEDULING AND BUDGETING
}

\author{
Remigiusz Romuald Iwańkowicz ${ }^{1}$ \\ 1 Faculty of Maritime Technology and Transport, West Pomeranian University of Technology, Al. Piastów 41, \\ 71-065 Szczecin, Poland, e-mail: iwankow@zut.edu.pl
}

Received: 2015.01.06

Accepted: 2015.02.10

Published: 2015.03.01

\begin{abstract}
The specificity of the yard work requires the particularly careful treatment of the issues of scheduling and budgeting in the production planning processes. The article presents the method of analysis of the assembly sequence taking into account the duration of individual activities and the demand for resources. A method of the critical path and resource budgeting were used. Modelling of the assembly was performed using the acyclic graphs. It has been shown that the assembly sequences can have very different feasible budget regions. The proposed model is applied to the assembly processes of large-scale welded structures, including the hulls of ships. The presented computational examples have a simulation character. They show the usefulness of the model and the possibility to use it in a variety of analyses.
\end{abstract}

Keywords: assembly sequence planning, schedulling, shipbuilding, budgeting, resource consumption, ship hull.

\section{INTRODUCTION}

Assembly is one of the most common operations in the production processes. It involves combining components in a separable and inseparable way. As a result, a complex product called the assembly is created. The specificity of the ship's structure is connected with a large number of elements [10]. The production of the ship's hull consists of a combination with welding methods in an inseparable way [13, 14]. The hull, as the final assembly, constitutes a unified whole. It is a distinctive feature differentiating the hull assembly from the mechanical assembly. In case of the shipbuilding process, the component may be the so-called subassembly, i.e. the structure assembled in advance (Figure 1).

The components can be assembled in a different order called a sequence. Each sequence of the assembly leads to the same result - the final structure. However, the sequences differ in terms of value of many criteria, for example: the difficulty of implementation, energy costs, safety of employees, the required qualifications of welders, the total execution time, etc. The selection of the best sequence is therefore a multi-objective optimisation problem. The whole class of this type of problems and methods of their solving is called the assembly sequence planning (ASP).

The ASP methods are developed mainly in the area of mechanical assembly, however, in recent years also the assembly of hulls becomes the subject of theoretical studies.

The article proposed the views on ASP considering the resource budgeting. The proposed approach clearly shows that every sequence of assembly is accompanied by the space of allowed schedules and budgets. Integration of planning the assembly sequence with the scheduling allows to take phenomenon into consideration as early as at the stage of designing the structure.

\section{DESCRIPTION OF THE PROBLEM}

Scheduling problems of the assembly processes of the seagoing ship hulls are included in the models of assembly sequence planning (ASP) in a 


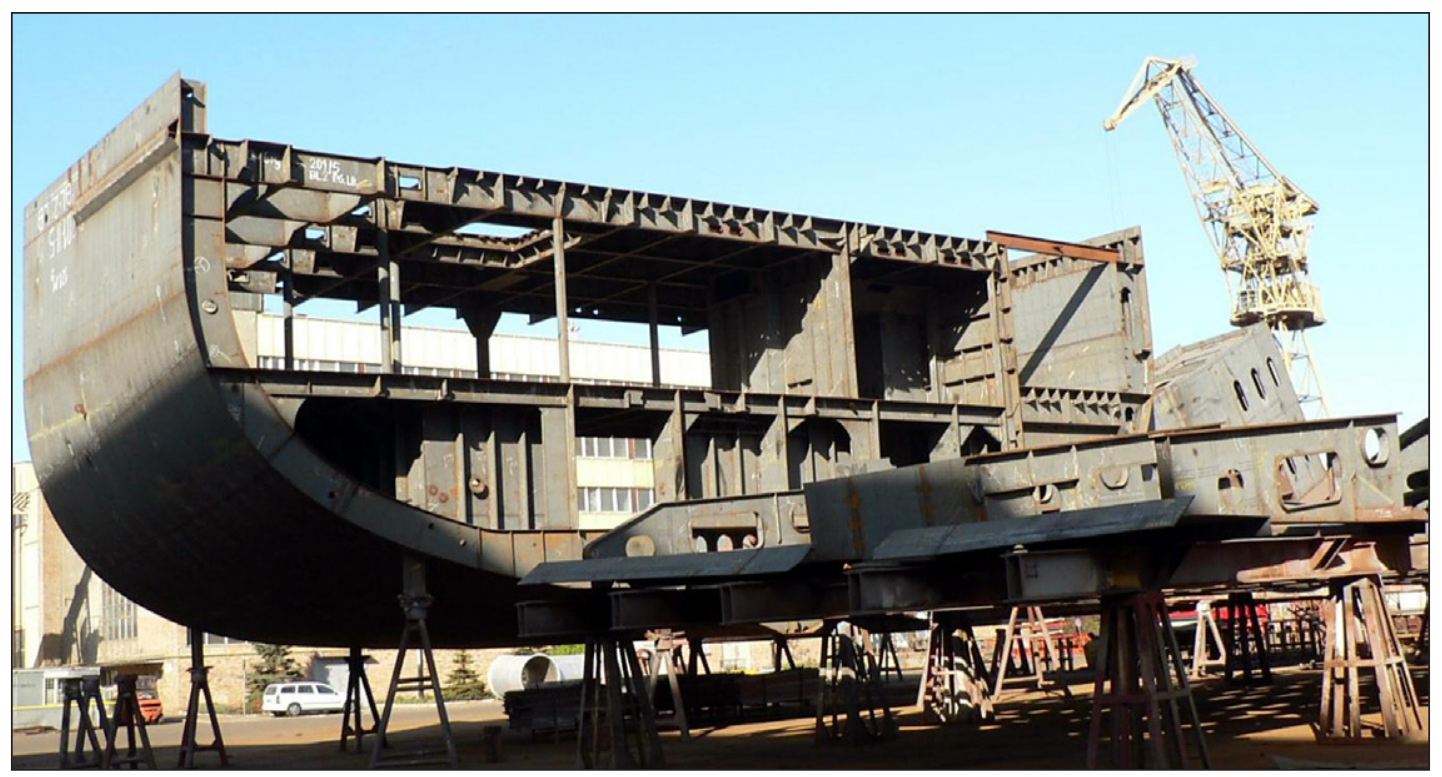

Fig. 1. Subassemblies of ship hull

simplified manner. The dominant approach here is the minimisation of the total time of the process.

The construction of the hull is a complex and lengthy process. This process is characterised by a variable intensity of work. There are three main stages: machining, prefabrication and final assembly of the hull. The prefabrication stage is characterised by the highest density. As a result, a typical graph of the labour budget of the hull's construction has the shape of a stretched letter $S$ [12]. Prefabrication and assembly of the hull mostly include the welding works [1]. In practice, not only the work as the resource is considered, that is a subject to budgeting. One should also take the resources of steel materials, welding materials, production area, and many other factors into consideration. Most often the planning of the new structure assembly takes place when the yard has already a fixed timetable of other works. This means that the demand for the resources is already fixed in a certain time perspective. The new assembly should be planned in order to take the existing timetable of works and the maximal intensity of the resource consumption into account, which the yard is able to secure.

\section{REVIEW OF THE LITERATURE}

For the construction of the hull so far the issue of ASP considering the resource budget has not been developed. Wei [15] considered the problem of outfitting the hull in terms of the distribution of workload over time. However, the issue of outfit- ting is more similar to the classical mechanical assembly and differs from the assembly of the welded structures.

Zhong., et al. [16] states that the methods of planning the hull assembly require a different approach. Models existing in this area are based on the analysis of construction in terms of connections and components occurring in it [5]. The considered criteria are mainly the similarity to the previously implemented solutions $[2,3,4,6$, $8,11,16]$. Authors very often propose the use of case based reasoning methods.

The general project budgeting problem on the basis of the fixed sequence was considered by Lin [7]. He proposed the method of generating the socalled feasible budget region (FBR) based on the critical path analysis.

In the assembly planning the acyclic graphs are used. Arcs of the graph are the organised neighbourhood relations between activities. For the fixed number of connections many assembly sequences can be generated. Robinson [9] provided the dependence of the number of directed acyclic graphs on the number of nodes. Here we can observe the effect of explosion of the number of solutions.

\section{FEASIBLE BUDGETS REGION OF FIXED ASSEMBLY SEQUENCE}

There are $n$ connections considered, which should be performed during the assembly process. Every connection is attributed with a sepa- 
rate action. For every $i$-th activity there is a determined time of performance $t_{i}$ and the amount of resources $r_{i}$ used in the time unit. For simplicity, it is assumed that both of these values are expressed in the whole numbers. The overall resource consumption during the assembly is:

$$
\operatorname{ORC}(\mathbf{t}, \mathbf{r})=\sum_{i=1}^{n} t_{i} \cdot r_{i}
$$

The sequence of assembly is presented as the acyclic directed graph $G$, whose nodes are the steps of the performed calls. In the further part of determination, the sequence and graph of the assembly are treated as clear.

It is important that the overall resource consumption (1) is constant and independent of the choice of assembly sequence. The collection of acyclic graphs with the fixed number of nodes $n$ is determined with $\Gamma(n)$.

For the fixed sequence $G \in \Gamma(n)$ and the fixed time vector $\mathbf{t}$, based on the analysis of the critical path, for each activity there can be determined the terms of:

- the earliest beginning $e b_{i}=e b_{i}(G, \mathbf{t})$,

- the earliest ending $e f_{i}=e f_{i}(G, \mathbf{t})$,

- the latest beginning $l b_{i}=l b_{i}(G, \mathbf{t})$,

- the latest ending $I f_{i}=I f_{i}(G, \mathbf{t})$.

Assuming that the assembly is started in the term 0 , the ending term of the process is as follows:

$$
t_{f}(G, \mathbf{t})=\max _{i=1, \ldots, n}\left\{l f_{i}(G, \mathbf{t})\right\}
$$

If at least one actions has a non-zero time supply $\left(l b_{i} \neq e b_{i}\right)$, then in the time period $\left[0, t_{f}\right]$ different schedules can be realised.

The set of allowable assembly schedules for the sequence $G$ and times $\mathbf{t}$ is defined as follows:

$$
\begin{gathered}
S(G, \mathbf{t})=\left\{\mathbf{s}=\left(s_{1}, \ldots, s_{n}\right) \mid \forall i=\right. \\
\left.=1, \ldots, n: e b_{i}(G, \mathbf{t}) \leq s_{i} \leq l b_{i}(G, \mathbf{t})\right\}
\end{gathered}
$$

where the schedule $\mathbf{s}$ is the vector of terms of beginning the activity. It can be observed that the number of all allowable schedules is:

$$
|S(G, \mathbf{t})|=\prod_{i=1}^{n}\left(l b_{i}(G, \mathbf{t})-e b_{i}(G, \mathbf{t})+1\right)
$$

For each fixed schedule $\mathbf{s}$ there is determined the use of resources in subsequent time units $1,2, \ldots, t_{f}$ :

$$
r c_{j}(G, \mathbf{t}, \mathbf{s}, \mathbf{r})=\sum_{\substack{i \in N(G, \mathbf{t}, \mathbf{s}, j) \\ \mathbf{s} \in S}} r_{i}, \quad j=1, \ldots, t_{f}(G, \mathbf{t}) ;
$$

where $N(G, \mathbf{t}, \mathbf{s}, j)$ is the set of indices of activities realised in the $j$-th time unit of the schedule $\mathbf{s}$.

The budget of the assembly is defined as the function of the schedule and time:

$B(G, \mathbf{t}, \mathbf{s}, \tau)=\sum_{j=1}^{\tau} r c_{j}(G, \mathbf{t}, \mathbf{s}, \mathbf{r}), \quad \tau=1, \ldots, t_{f}(G, \mathbf{t})$

At the beginning of the process, the use of the resource is zero. Therefore, it is assumed:

$$
\forall \mathbf{s}: \quad B(G, \mathbf{t}, \mathbf{s}, 0)=0
$$

The budget determines how the use of the determined amount is used in time $\operatorname{ORC}(\mathbf{t}, \mathbf{r})$ (see equation (1)). It can be seen that for the final term: $B\left(G, \mathbf{t}, \mathbf{s}, t_{f}\right)=O R C(\mathbf{t}, \mathbf{r})$ for any $\mathbf{s}$. The earliest budget of the sequence is the one in which all connections are used the earliest it is possible $\left(\mathbf{s}=\left(e b_{1}, \ldots, e b_{n}\right)\right)$. Similarly, the latest budget of the sequence is the one in which $\mathbf{s}=\left(l b_{1}\right.$ $\left., \ldots, l b_{n}\right)$. Lin [7] calls the area included between the graphs of these budgets the feasible budgets region (FBR).

The way of determining FBR of the assembly sequence is presented in example 5 of the connections and the sequences presented in Figure 2.

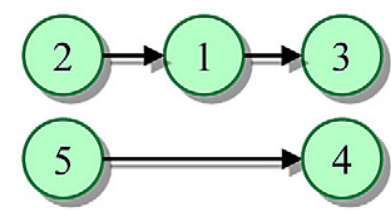

Fig. 2. An exemplary assembly sequence for 5 connections

Let the execution times and the required resources be as follows: $\mathbf{t}=(7,5,9,1,12), \mathbf{r}=(10$, $6,9,8,7)$. The overall consumption of resources during the assembly is: $\operatorname{ORC}(\mathbf{t}, \mathbf{r})=7 \cdot 10+5 \cdot 6+$ $9 \cdot 9+1 \cdot 8+12 \cdot 7=273$.

Based on the determined earliest and latest terms it can be observed that the beginning of the performance of the connection no. 4 can take place in 9 different terms: $12,13, \ldots, 20$. Similarly, connection 5 can be started in 9 terms: 0 , $1, \ldots$, 8. Therefore, there is considered $9 \cdot 9=81$ variants of schedules. Each variant has a different resource budget. Figure 3 presents 2 budgets creating FBR and 3 intermediate budgets.

It can be observed that even such a simplified assembly sequence (only 5 connections) allows to generate a diverse set of schedules and budgets.

Attention should be paid to the fact that for the determined amount of connections there can be 
Table 1. Results of the analysis of the critical path for the exemplary sequence

\begin{tabular}{|c|c|c|c|c|}
\hline \multirow{2}{*}{$\begin{array}{c}\text { Connection } \\
\text { /activity } \\
i\end{array}$} & \multicolumn{2}{|c|}{ Earliest terms } & \multicolumn{2}{|c|}{ Latest terms } \\
\hline & $\begin{array}{l}\text { beginning } \\
e b_{i}\end{array}$ & $\begin{array}{c}\text { ending } \\
\text { ef }\end{array}$ & $\begin{array}{l}\text { beginning } \\
\qquad \mathrm{lb}_{i}\end{array}$ & $\begin{array}{c}\text { ending } \\
\text { If }_{i}\end{array}$ \\
\hline 1 & 5 & 12 & 5 & 12 \\
\hline 2 & 0 & 5 & 0 & 5 \\
\hline 3 & 12 & 21 & 12 & 21 \\
\hline 4 & 12 & 13 & 20 & 21 \\
\hline 5 & 0 & 12 & 8 & 20 \\
\hline
\end{tabular}

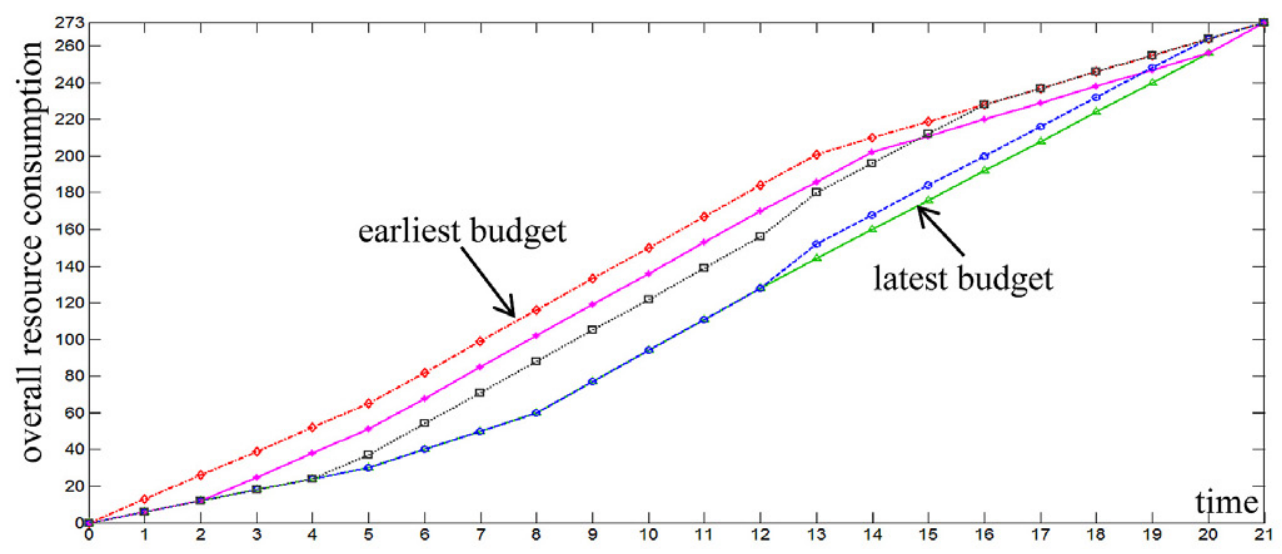

Fig. 3. FBR and the selected allowable budgets for the considered assembly sequence

generated many assembly sequences, which differ in the FBR shape. For the determined durations and times of using the resources all sequences have the same determined value $\operatorname{ORC}(\mathbf{t}, \mathbf{r})$. This means that the height of the FBR is the same. However, these regions may differ in the dimension along the time axis, what results directly from differences in the duration of the sequence variants. This will be illustrated by the example of three assembly sequences for 5 connections (Figure 4).
It is assumed that the time vector $\mathbf{t}$ and the resource vector $\mathbf{r}$ are the same as in the previous example. FBR of sequence 1 was shown in Figure 3 . For the sequence 2 and 3 the terms determined with the critical path method are presented in Tables 2 and 3. It is visible that three basic variants of the assembly sequence differ in the final terms. These are, respectively: 21,26 and 28. FBR for the considered three assembly sequences are presented in Figure 5. sequence 1:

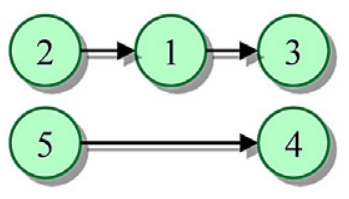

sequence 2:

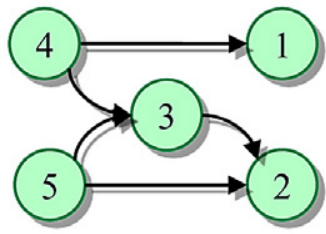

sequence 3:

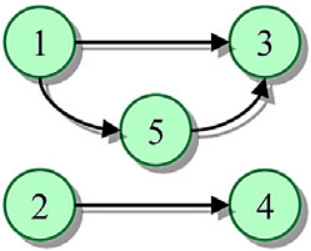

Fig. 4. Exemplary assembly sequences for 5 connections

Table 2. Results of the critical path analysis for sequence 2

\begin{tabular}{|c|c|c|c|c|}
\hline \multirow{2}{*}{$\begin{array}{c}\text { Connection } \\
\text { /activity }\end{array}$} & \multicolumn{2}{|c|}{ Earliest terms } & \multicolumn{2}{c|}{ Latest terms } \\
\cline { 2 - 5 } & $\begin{array}{c}\text { beginning } \\
e b\end{array}$ & $\begin{array}{c}\text { ending } \\
\text { ef }\end{array}$ & $\begin{array}{c}\text { ending } \\
\text { If }\end{array}$ \\
\hline 1 & 1 & 8 & 19 & $\mathbf{2 6}$ \\
\hline 2 & 21 & 26 & 21 & $\mathbf{2 6}$ \\
\hline 3 & 12 & 21 & 12 & 21 \\
\hline 4 & 0 & 1 & 11 & 12 \\
\hline 5 & 0 & 12 & 0 & 12 \\
\hline
\end{tabular}


Table 3. Results of the critical path analysis for sequence 3

\begin{tabular}{|c|c|c|c|c|}
\hline \multirow{2}{*}{$\begin{array}{l}\text { Connection } \\
\text { /activity }\end{array}$} & \multicolumn{2}{|c|}{ Earliest terms } & \multicolumn{2}{|c|}{ Latest terms } \\
\hline & $\begin{array}{c}\text { beginning } \\
e b\end{array}$ & $\begin{array}{c}\text { ending } \\
\text { ef }\end{array}$ & $\begin{array}{c}\text { beginning } \\
\mathrm{lb}\end{array}$ & $\begin{array}{l}\text { ending } \\
\text { If }\end{array}$ \\
\hline 1 & 0 & 7 & 0 & 7 \\
\hline 2 & 0 & 5 & 22 & 27 \\
\hline 3 & 19 & 28 & 19 & 28 \\
\hline 4 & 5 & 6 & 27 & 28 \\
\hline 5 & 7 & 19 & 7 & 19 \\
\hline
\end{tabular}

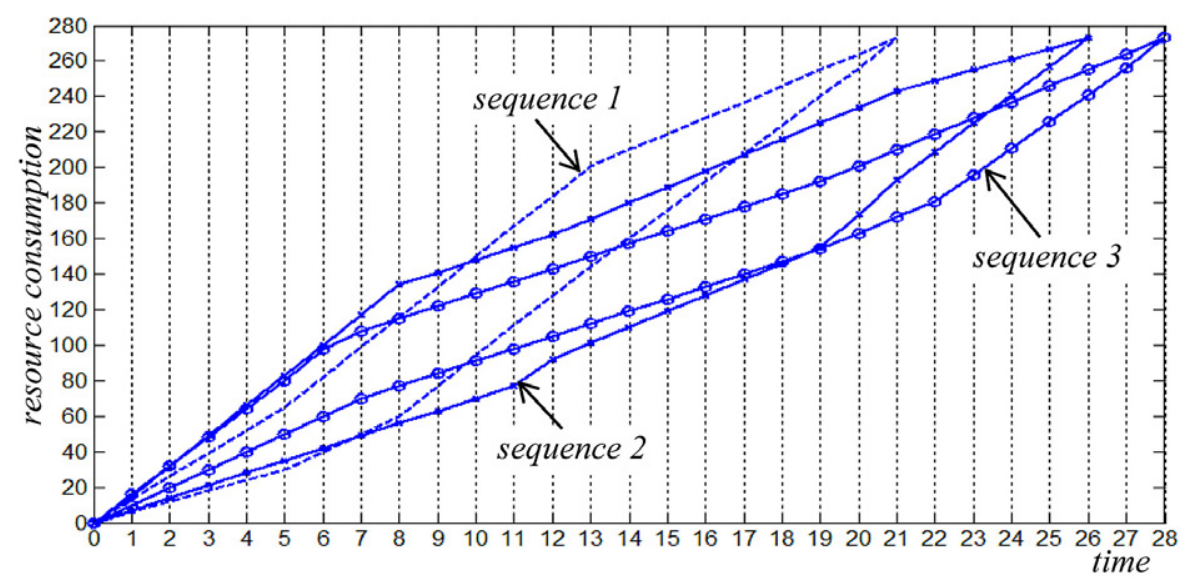

Fig. 5. Feasible budget regions for three exemplary assembly sequences

Based on the analysis of feasible budgets regions it can be observed: differences in the duration of the process and in the available scope of control of the speed of the resource consumption. Sequence 2 in every time unit has the widest scope and as a result the greatest FBR surface.

\section{CRITERIA FOR THE SELECTION AND LIMITATION OF THE ASSEMBLY SEQUENCE}

The network model and the FBR of the every considered assembly sequence variant allows to implement limitations and criteria to the ASP process. From the point of view of the work organisation of the production system it is important to consider the level of available production capacities of the system. They are inherently limited. The level of the free production capacity can be decreased by simultaneous implementation of other processes. One should also take into consideration the fact that the excess of the contracted completion term of the construction to the client or the co-operating party may result in the financial penalties or disorganisation of the production system work.

The peak resource consumption and the duration of the process are the basic measures char- acterising the assembly sequence. The time of the process $t_{f}(G, \mathbf{t})$ (see equation) is the number clearly defined for a given sequence $G$. The peak resource consumption $r C_{\text {peak }}(G, \mathbf{t}, \mathbf{r})$ requires the analysis of all schedules $\mathbf{s} \in S(G, \mathbf{t})$ and search for the variant with the lowest peak (see equation (5)):

$$
r c_{\text {peak }}(G, \mathbf{t}, \mathbf{r})=\min _{\mathbf{s} \in S(G, \mathbf{t})}\left\{\max _{j=1, \ldots, t_{f}(G, \mathbf{t})}\left\{r c_{j}(G, \mathbf{t}, \mathbf{s}, \mathbf{r})\right\}\right\}
$$

The sequence analysis for 5 connections was conducted for the data similar to the ones in chapter $4: \mathbf{t}=(7,5,9,1,12), \mathbf{r}=(10,6,9,8,7)$. There was generated the whole set $\Gamma(n)$, and then for every sequence there was determined:

- time of realisation of the assembly process $t_{p}$

- all possible schedules and their corresponding peak resource consumption $r C_{\text {peak }}$,

- schedule with the lowest peak consumption.

Consequently, a 29 281-element set $\Gamma(n)$ was obtained. Figure 6 presents the durations of all sequences.

It is visible that for the adopted data the assembly can last from 12 to 34 time units. There are no sequences lasting for $23,30,31$ and 32 time units. Sequences with the longest time are serial. Their time is the sum of the times of all activities. Sequences with the shortest time have 


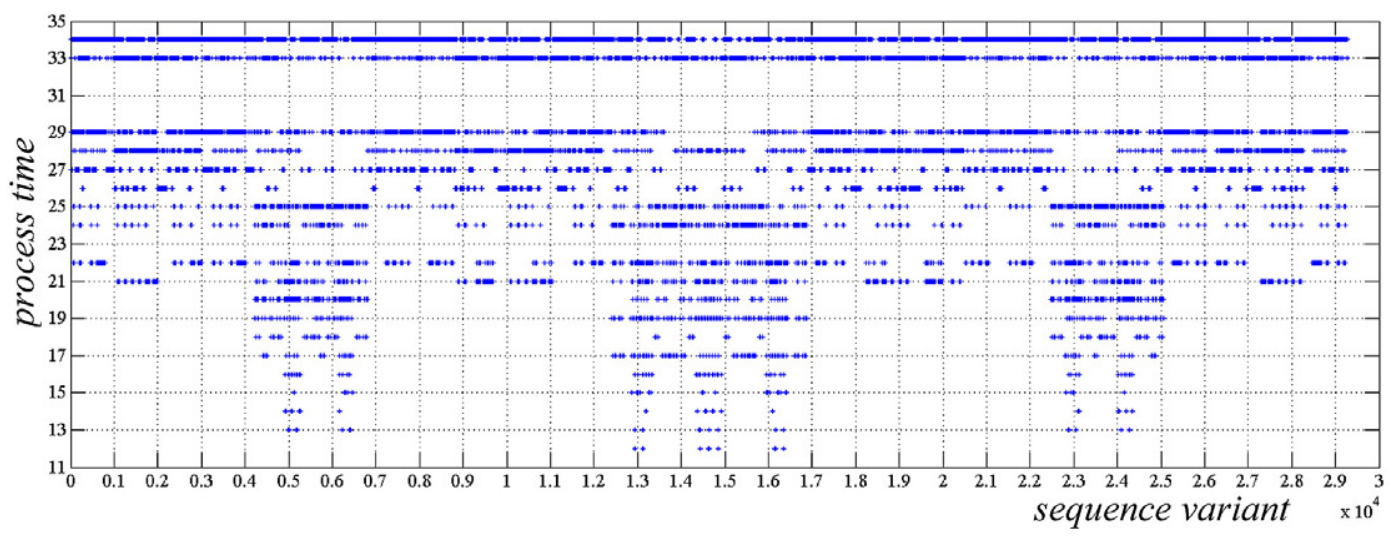

Fig. 6. Durations of the generated assembly sequences

the parallel character. All others are realised during the longest activity.

Figure 7 presents the peak levels of the resource consumption achievable for every sequence. The peak resource consumption fits into the range [10, 32]. The highest peak values (32) have 4 sequences shown in Figure 8. The lowest level of the peak consumption is equal to the demand of the most resource-absorbent activity.

Figure 9 presents the dependency between the studied variables. It can be observed that the total number of different variants equals 55. It is a very small value compared to the number of the analysed sequences. As expected, it can be observed that all serial sequences, with the longest duration, have the same peak resource consumption level. Sequences with the highest peak consumption, shown in Figure 8, have the same duration. It is the lowest and equals 12 . Also other sequences have the same duration, which peak resource consumption is lower.

In the figure the points creating the paretooptimal set have been marked due to the criteria

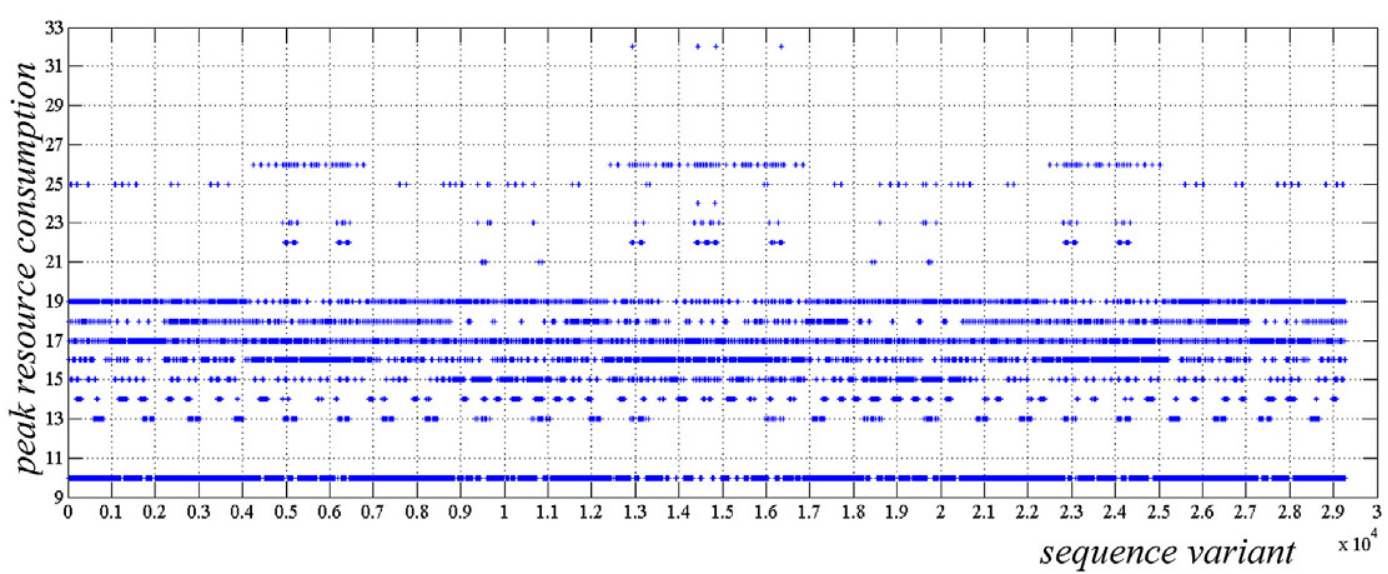

Fig. 7. Peak resources consumption for the generated assembly sequences
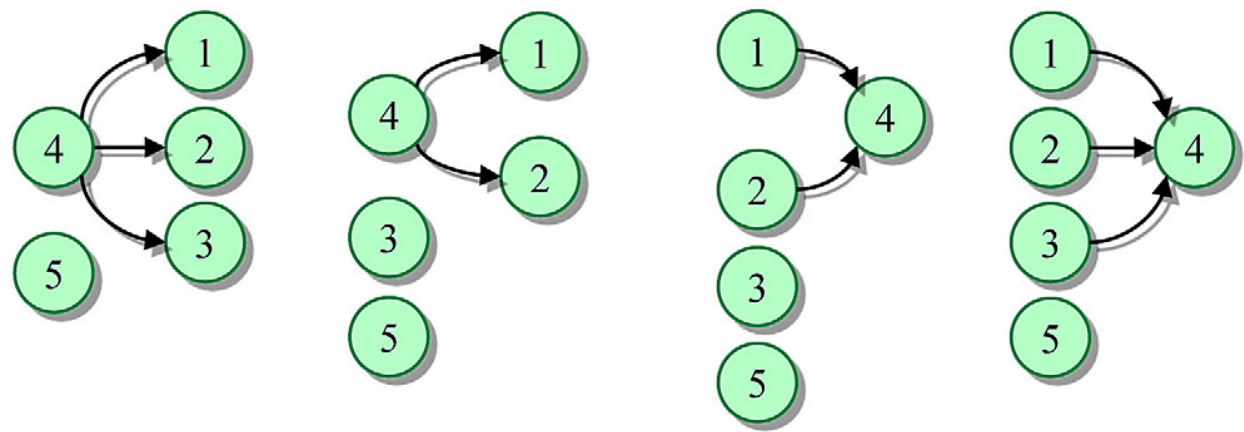

Fig. 8. Sequences with the highest peak resource consumption 


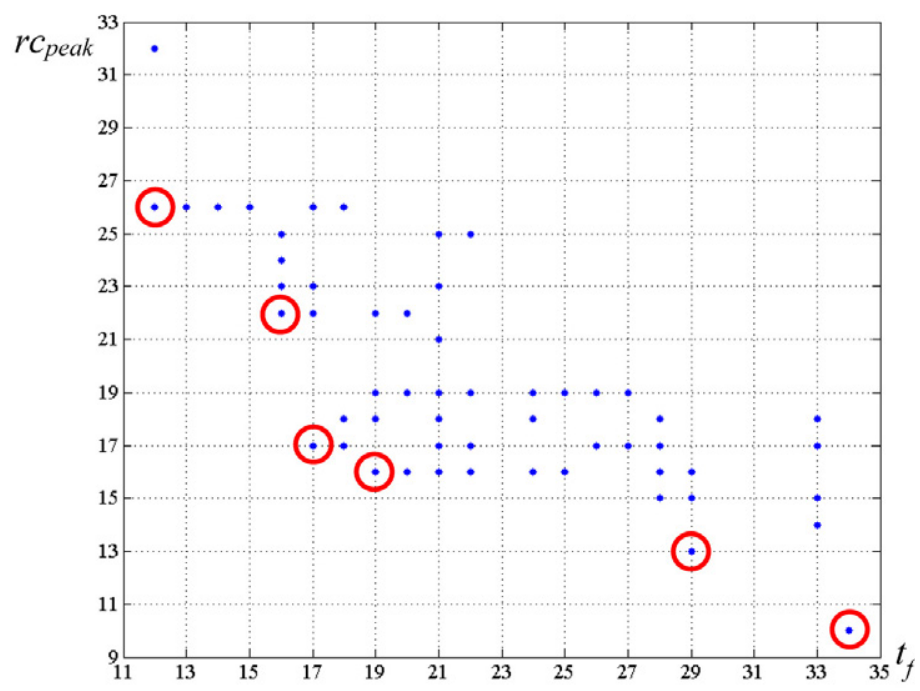

Fig. 9. Dependence of the peak resource consumption level on the process time

of time minimisation and the peak resource consumption. In many cases this set is searched in order to find a rational solution.

Time boundary values of the assembly completion $t_{F}$ and the peak resource consumption $r c_{\text {Peak }}$ can be adopted as the parameters dividing the set $\Gamma(n)$ into subsets of the acceptable and unacceptable sequences. As a result two conditions are obtained, which must be met by the acceptable sequence $G$ with the fixed vectors $\mathbf{t}$ and $\mathbf{r}$ :

$$
\begin{gathered}
t_{f}(G, \mathbf{t}) \leq t_{F} \\
r c_{\text {peak }}(G, \mathbf{t}, \mathbf{r}) \leq r c_{\text {Peak }}
\end{gathered}
$$

For the studied case 5 connections determined the number of the acceptable sequences depending on the values of the limiting parameters $t_{F}$ and $r c_{\text {Peak }}$ (Figure 10).

The analysis of the number of the set of the acceptable sequences may be significant, when during the planning of the assembly sequences other criteria and limitations not connected with the schedule are also considered. Insufficient sequences acceptable at the stage of the schedule analysis may lead to an empty set of solutions after considering other limitations. On the other hand, a very large set of solutions will require the use of the randomised methods of search and the long computation time.

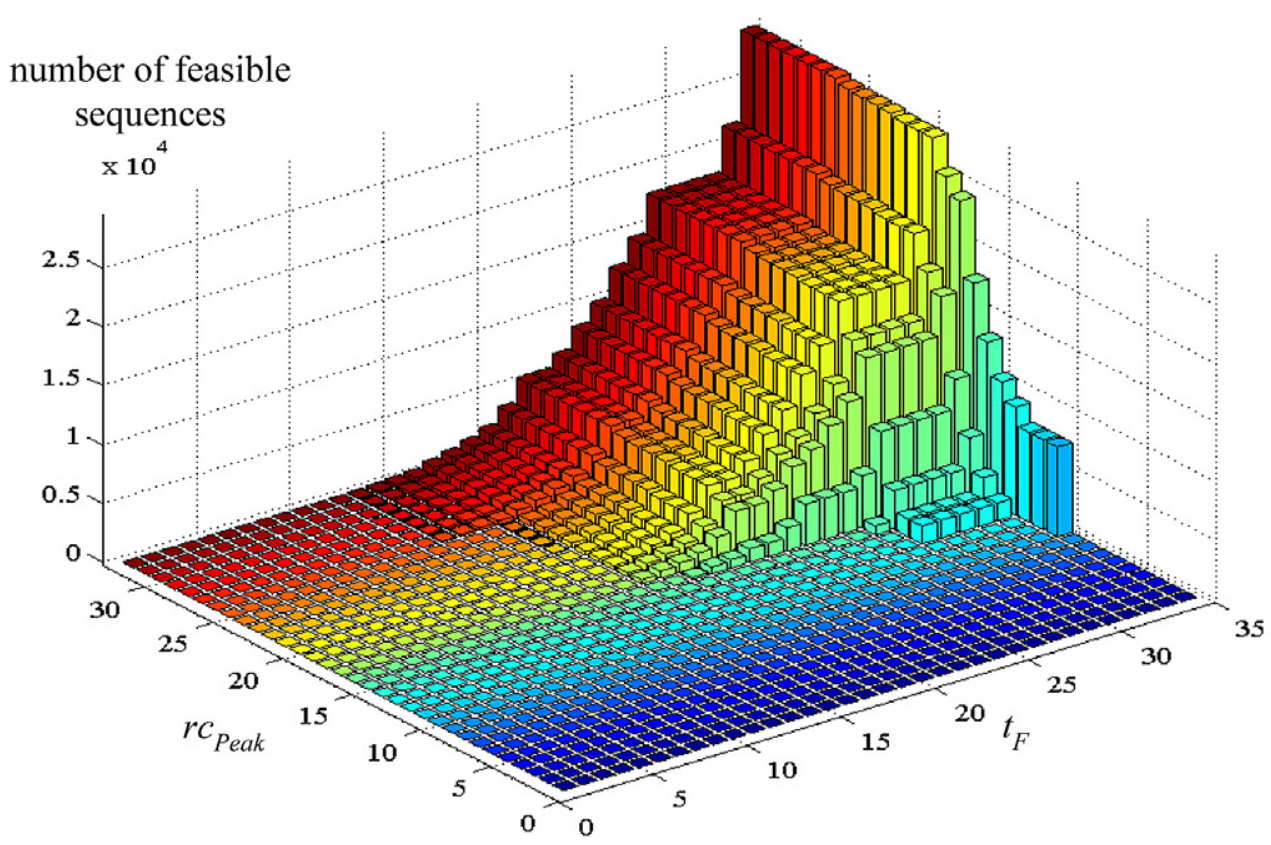

Fig. 10. The number of acceptable sequences depending on the limitation values 


\section{CONCLUSIONS}

The presented theoretical model allows to account for scheduling issues and budgeting during planning of the assembly sequences. Such an approach is necessary in case of the assembly of large-scale structures. This type of processes are long and consume significant amounts of resources. Yards planning the production must synchronise many assembly processes implemented at the same time. Resource budget management in time is therefore crucial for them.

The proposed method to determine the peak resource consumption for the determined assembly sequence of course requires the use of a computer. The analysis should be performed on a huge amount of variants, which is strongly increasing with the increase of the number of connections. In practice, the shipbuilding number of the analysed connections is reduced by the use of the subassembly.

Two approaches to the assembly scheduling analysis were shown. In the first one, the process time and the peak resource consumption are the basis for the formulation of optimisation criteria. The way of determining the pareto-optimal solutions was shown. In the second approach, conditions limiting the set of acceptable solutions are formulated due to the time and consumption of the resource.

The examples of the calculations were conducted for 5 connections and randomly selected vectors of data. Vast possibilities of the assembly sequence analysis in terms of the schedule and resources budget were presented.

\section{REFERENCES}

1. Batista H.S., Graczyk T., An analysis of the welding-induced deformation of ship panels using a statistical tool. Polish Maritime Research, 20 (2), 2013, 74-81.

2. Chi Z., Jun S., Intelligentized work-preparation for ship hull construction with Optimized Assembly Planning System. Proceedings of the IEEE International Conference on Systems, Man and Cybernetics, Istanbul, Turkey, 10-13 October 2010, 2740-2744.
3. Cho K.-K., Lee S.-H., Chung D.-S., An automatic process-planning system for block assembly in shipbuilding. Annals of the CIRP, 45 (1), 1996, 41-44.

4. Cho K.-K., Sun J.-G., Oh J.-S., An automated welding operation planning system for block assembly in shipbuilding. International Journal of Production Economics, 60-61, 1999, 203-209.

5. Iwańkowicz R., Optimization of assembly plan for large offshore structures. Advances in Science and Technology Research Journal, 6(16), 2012, 31-36.

6. Iwańkowicz R., Application of multi-dimensional grouping to building steel stiffened shell structures. Polish Maritime Research Vol. 20, 1(77), 2013, 48-58.

7. Lin E.Y.H., The role of the activity adjacency matrix in the critical path method. APL Quote Quad, 21 (3), 1991, pp. 1419.

8. Qu S., Jiang Z., Tao N., An integrated method for block assembly sequence planning in shipbuilding, The International Journal of Advanced Manufacturing Technology, 69 (5-8), 2013, 1123-1135.

9. Robinson R.W., Counting labeled acyclic digraphs. [In:] New Directions in Graph Theory (Ed. F. Harary). Academic Press, New York 1973.

10. Sekulski Z, Ship hull structural multiobjective optimization by evolutionary algorithm. Journal of Ship Research, 58 (2), 2014), 45-69.

11. Seo Y., Sheen D., Kim T. , Block assembly planning in shipbuilding using case-based reasoning. Expert Systems with Applications, 32, 2007, 245-253.

12. Storch R.L., Hammon C.P., Bunch H.M., Moore R.C., Ship production, 2nd ed. Cornell Maritime Press, Centreville, Maryland 1995.

13. Urbański T., The influence of welding deformation types of hybrid node on assembly suitability. Advances in Science and Technology Research Journal, 6 (16), 2012, 42-47.

14. Urbański T., Taczała M., Experimental investigation of deformations of hybrid structural joint. [In:] 4th International Conference on Maritime Structures (MARSTRUCT 2013), Espoo, Finland, 2527 March 2013, Taylor\&Francis Group, London 2013, 441-446.

15. Wei Y., Automatic generation of assembly sequence for the planning of outfitting processes in shipbuilding [Ph.D. thesis]. VSSD, Delft, the Netherlands 2012.

16. Zhong Y., Xue K., Shi D., Assembly unit partitioning for hull structure in shipbuilding. ComputerAided Design, 45 (12), 2013, 1630-1638. 